\title{
Computational model for particle deposition in turbulent gas flows for CFD codes
}

\author{
M. C. Paz, J. Porteiro, A. Eirís \& E. Suárez \\ CFD Simulation Group, University of Vigo, Spain
}

\begin{abstract}
A numerical simulation procedure was developed in FLUENT code in order to study particle deposition in diluted turbulent gas flows in ducts. A hybrid Eulerian-Lagrangian model was used to evaluate the gas flow and temperature profile as well as particle dispersion and deposition. The mean flow velocity and temperature fields were generated by a Reynolds Average Navier Stokes (RANS) method, using the Reynolds Stress Model (RSM) available in FLUENT. The instantaneous turbulent velocity fluctuation and Brownian force were simulated by a continuous Gaussian random field as a white-noise process respectively. In the particle equation of motion, Brownian diffusion, Saffman lift, Stokes drag and gravity were included. In order to evaluate the deposition model, particles with diameter ranges from $1 \mathrm{~nm}$ to $50 \mu \mathrm{m}$, and different velocity fields were studied. The numerical results obtained under isothermal conditions were compared with the available experimental data, with empirical correlations, and earlier published simulation results. In all cases, an acceptable agreement was observed. The importance of each of the deposition forces included is discussed in this work.

The reasonable computational cost of this methodology enables us to study particle deposition in complex geometries, different from smooth surfaces. The results presented provide guidelines to develop an extended computational fouling model in heat exchange surfaces.
\end{abstract}

Keywords: particles deposition, exhaust gas, fouling, CFD.

\section{Introduction}

Particle deposition is of critical importance in numerous industrial applications, filtration, microchip manufacture, atmospheric pollutant control, combustion and 
chemical processes. Moreover it is essential in heat exchanger fouling phenomena [1]. Often these deposition particles processes involve turbulent and complex geometry which explain that Computational Fluid Dynamics (CFD) codes are more frequently used in the modeling of this process. So, to extend the capability of the codes to include the deposition of particles, including turbulent flow field effects as easily as possible, is a logical step.

The object of the current research is to develop a simple computational particle deposition methodology with good accuracy in predicted particle deposition rate in turbulent duct flows. This tool was implemented in a customised version of the commercial CFD software package FLUENT 6.3.26. The advantage of this model is its simplicity and computational efficiency.

\section{Particle deposition basics}

The deposition of soot particles in gas turbulent flows is a subcategory of twophase flows with a particle load which is usually very low. Therefore interaction between phases is unidirectional, i.e. continuum fluid phase (air) affects the dispersed solid phase (soot particles), and not in the other direction. In this study, it is assumed that the flow is sufficiently dilute or dispersed. Mean distance between particles is big enough to be considered, so we focused on isolated particles and particulates collisions were ignored. Therefore the continuous phase was resolved using Eulerian description, and the particle phase was resolved with Lagrangian approach.

There are different approaches for the treatment of the interaction between particles and the continuous phase.

The choice of Euler or Lagrange models for each phase was selected taking into account two parameters; particle load $\beta$ eqn (1) and the mean distance between particles defined by Crowe et al. [2].

The Stokes number is a measure of coupling between dispersed and continuous phases, eqn (1). The particle relaxation time which is dimensionless with wall units, $\tau^{+}$, is a specific Stokes number and is a relation between the response time of the particle and the characteristic time of the turbulent structures of the wall, eqn (2). It is a measurement of the capability of the particles to follow the turbulent fluid structures near the wall. The higher the time, the less affected the particles are by the flow and for low values, particles follow the flow.

$$
\begin{gathered}
\beta=\frac{\alpha_{p} \rho_{p}}{\alpha_{f} \rho_{f}} \quad, \quad S t=\frac{\tau_{p}}{\tau_{f}} \\
\tau_{p}=\frac{\rho_{p} \cdot d_{p}^{2}}{18 \cdot \mu} \quad \tau_{f}=\frac{v}{u^{* 2}} \quad \tau^{+}=\frac{\tau_{p}}{\tau_{e}}=\frac{\rho_{p} \cdot d_{p}^{2} \cdot \rho \cdot u^{* 2}}{18 \cdot \mu^{2}}
\end{gathered}
$$

There is a strong relation between the deposition velocity and the capability of the particles to follow the fluid flow (dimensionless relaxation time). Three different regimes can be defined, according to the size of particles; diffusion, inertia and impaction. 
At present the deposition processes may be studied from three different approaches; experimental research, phenomenological and numerical models. Most published papers about fouling are focused on experimental research, Fuchs [3], Liu and Agarwal [4], Wood [5], Hinds [6], and Papavergos and Hedley [7] show empiric expressions for each of the three deposition particles regimes. As is known, particle deposition is one of the most important mechanisms involved in fouling. Phenomenological models have been developed to predict fouling layer evolution; the most remarkable could be Epstein [8].

The Euler-Lagrange models are divided taking into account the turbulent treatment used in the fluid phase usually in RANS-, LES- and DNS-Lagrange models. One of the most prolific research methods for analysing particle transport, dispersion and deposition is the RANS-Lagrange model approach, which is used in this work.

\section{Computational model}

\subsection{Mean flow field}

The interaction of particle-fluid determines the particle deposition rate, hence the solution of the mean flow field is critical for the later solution of the dispersed phase. The continuous gaseous phase was resolved without being affected by the particles. To keep the simplicity of the model, the Eulerian approach was chosen. The RANS-based modelling approach and the segregated solver were adopted. The viscosity affected near wall region was resolved by enhanced near-wall treatment using the Reynolds Stress Model RSM, incorporated in the Fluent code [9].

It is well known that in a turbulent flow field, the effect of instantaneous flow fluctuation is the main mechanism for particle deposition [10]. When a particle goes through a turbulent flow field, it interacts with eddies successively. It is caught by an eddy for a time before being affected by another eddy, so it is important to determine the instantaneous velocity of an eddy, the time that the particle takes to cross the eddy, the eddy life time, the particle size and the eddy size.

Therefore, it is fundamental to characterize the turbulence fluctuations as well as possible and RSM is the most complete and suitable model for this performance of the RANS models, although it involves a bigger computational effort. As this model is not based on the Boussinesq hypothesis, anisotropy turbulence effects are taken into account, so the instantaneous fluid velocity is simulated given the local mean velocity and therefore, the fluctuating velocity component is calculated with the mean square root of local fluctuation velocity in $i$ direction, eqn (3). Hence, different results are obtained for each direction, in contrast with the rest of the RANS models. This discrete random walk model requires the calculation of several particle "attempts" in order to obtain representative results.

$$
u_{f, i}=\overline{u_{f, i}}+u_{f, i}^{\prime} \quad, \quad u_{f, i}^{\prime}=G \sqrt{\overline{u_{f, i}^{\prime 2}}}
$$


The interaction time, $t_{i}$, is given by eqn (4), where $\tau_{e}$ is the eddy life time, defined with the particle Lagrangian integral time scale $T_{L}$, that for RSM model, $T_{L} \approx 0.3 \mathrm{k} / \varepsilon$ and $\tau_{\text {cross }}$ is the eddy cross time of a particle.

$$
\begin{gathered}
t_{i}=\min \left(\tau_{e}, \tau_{\text {cross }}\right) \\
\tau_{e}=2 T_{L} \quad, \quad \tau_{\text {cross }}=-\tau_{p} \ln \left[1-\left(\frac{L_{e}}{\tau_{p}\left|u_{f}-u_{p}\right|}\right)\right]
\end{gathered}
$$

\subsection{Dispersed phase}

The multiphase model, Discrete Phase Module DPM, included in Fluent was used to simulate the solid phase. This multiphase submodel calculates the particles motion using the Lagrangian method. This method tracks particles solving the force balance based on the predicted continuous phase flow field. Eqn (5) is the governing equation in $i$ direction, $m_{p}$ is the particle mass, $u_{p, i}$ is the particle velocity component in $i$ direction and $\Sigma F_{i}$, is the resultant force applied to the particle in the same direction.

$$
m_{p} \frac{d u_{p, i}}{d t}=\sum F_{i}
$$

Forces affecting the particles are: Fluid dynamics (drag, Magnus, Saffman lift, turbophoresis, Basset and virtual mass), static force (buoyancy), external forces (gravity, electromagnetic and electrostatic) and molecular forces (Brownian and thermophoresis).

When the density of the particles is much bigger than the density of the gas, as in this case, Basset, virtual mass, Magnus effect and buoyancy can be ignored.

\subsubsection{Drag force}

Drag force modifies the particle path in the sense of reducing the slip between particle and fluid. The drag force is expressed as a function of $C_{D}$, the dimensionless drag coefficient. Several expressions have been developed for different Reynolds ranges [6]. In this study the drag coefficient is computed with the adjusted expression of the particle Reynolds number $\operatorname{Re}_{p}$, eqn (6) given by Morsi and Alexander [11], where $a_{\mathrm{s}}$ coefficients are constants for spherical particles.

$$
C_{D}=a_{1}+\frac{a_{2}}{\operatorname{Re}_{p}}+\frac{a_{3}}{\operatorname{Re}_{p}^{2}}, \operatorname{Re}_{p}=\frac{\rho\left(u_{f}-u_{p}\right) d_{p}}{\mu}
$$

If the particle size is of a similar order of magnitude as the mean free path of the continuum phase $\lambda$, a drag correction factor should be included, the StokesCunningham slip correction given in eqn (7), as a Knudsen number $(\mathrm{Kn})$ function.

$$
C_{c}=1+\frac{2 \lambda}{d_{p}}\left(1.257+0.4 e^{1.1 d_{p} / 2 \lambda}\right) \quad, \quad K n=2 \frac{\lambda}{d_{p}} \quad, \quad \lambda=2 v \sqrt{\frac{\pi M}{R T}}
$$




\subsubsection{Saffman lift}

The Saffman lift force is not usually taken into account for submicron particles but with a high shear field, as occurs in the vicinity of the wall, this becomes more important. The original expression [12] was extended for tridimensional flow, with the deformation rate tensor $s_{i j}$ [13], eqn (8), where $K=2.594$, is the coefficient of Saffman' lift force.

$$
\frac{F_{\text {Saffman }, j}}{m_{p}}=\frac{2 K \cdot v^{1 / 2} s_{i j}}{\frac{\rho_{p}}{\rho}\left(s_{l k} s_{k l}\right)^{1 / 4}}\left(u_{f, j}-u_{p, j}\right) \quad, \quad s_{i j}=\frac{1}{2}\left(\frac{\partial u_{i}}{\partial x_{j}}+\frac{\partial u_{j}}{\partial x_{i}}\right)
$$

\subsubsection{Turbophoresis}

The turbophoretic effect is the resultant force due to the anisotropic turbulence intensity, which is bigger near the walls. Several researchers emphasize its importance in deposition processes [13]. It has to be included in the Eulerian framework, but in a Lagrangian framework, the instantaneous equation of the particle path is resolved, so the random component of the velocity is taken into account and therefore, the turbophoretic effects are implicitly included.

\subsubsection{Brownian diffusion}

Brownian diffusion is modeled through the Li and Ahmadi expressions [13], eqn (9), where $\zeta_{j}$ is a Gaussian white noise random process with zero mean and unit variance, $K_{B}$ is the Stefan-Bolzmann constant, $\Delta \mathrm{t}$ is the time step for particle tracking, and $T$ is the absolute Temperature of the gas. Brownian force strongly increases when $d_{p}$ decreases, so it is an important mechanism near the wall for small particles.

$$
F_{\text {Brownian }, j}=\zeta_{j} \cdot m_{p} \sqrt{\frac{\pi S_{0}}{\Delta t}} \quad, \quad S_{0}=\frac{216 v K_{B} T}{\pi^{2} \rho d_{p}^{5}\left(\frac{\rho}{\rho_{p}}\right)^{2} C_{c}}
$$

\section{Results and discussion}

The duct shown in Figure 1 with a ten diameters previous zone to ensure fully developed conditions was used to reproduce the experimental conditions.

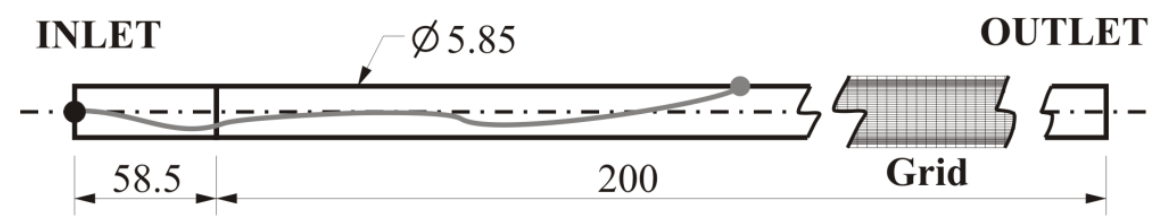

Figure 1: $\quad$ Scheme of simulation domain.

The geometry and the mesh of the tested tubes were generated using the Fluent code mesher, GAMBIT ${ }^{\mathrm{tm}}$. A structured two-dimensional mesh was made, adjusting the first cell size to the $y^{+}$required value and a total of 80000 cells were created. 
Particles were uniformly distributed in inlet. The stochastic tracking sub model was used to simulate the turbulence dispersion using 100000 attempts, which is high enough to ensure a representative value and this did not change by increasing the number of attempts.

The mass flow deposited was measured directly counting the particles deposited each time step and deposition velocity was estimated with area deposition and mean particle concentration $C_{p}=10^{-3} \mathrm{~kg} / \mathrm{m}^{3}$. The particle diameter range $d_{p}(1 \mathrm{~nm}$ to $50 \mu \mathrm{m})$ allows the coverage of all deposition regimes. Removal, detachment and sticking probability were not contemplated in this study, so the boundary conditions at walls were total adhesion, which is normal practice in these cases.

\subsection{Continuous phase}

The gas used in the simulations was air, with a temperature of $363.15 \mathrm{~K}$ and a mass flow rate of $0.0005 \mathrm{~kg} / \mathrm{s}$, with the viscosity computed by Sutherland law and the gas was considered as ideal and incompressible. The aim of the mean flow field simulation was to obtain the most realistic velocity field possible, and moreover to capture the anisotropy of the turbulence by means of the fluctuating velocity.

If dimensionless fluctuating velocity components, $u_{i}{ }_{i}$ are plotted as a function of wall dimensionless distance $y^{+}$the results show a good agreement with experimental results (Figure 2).

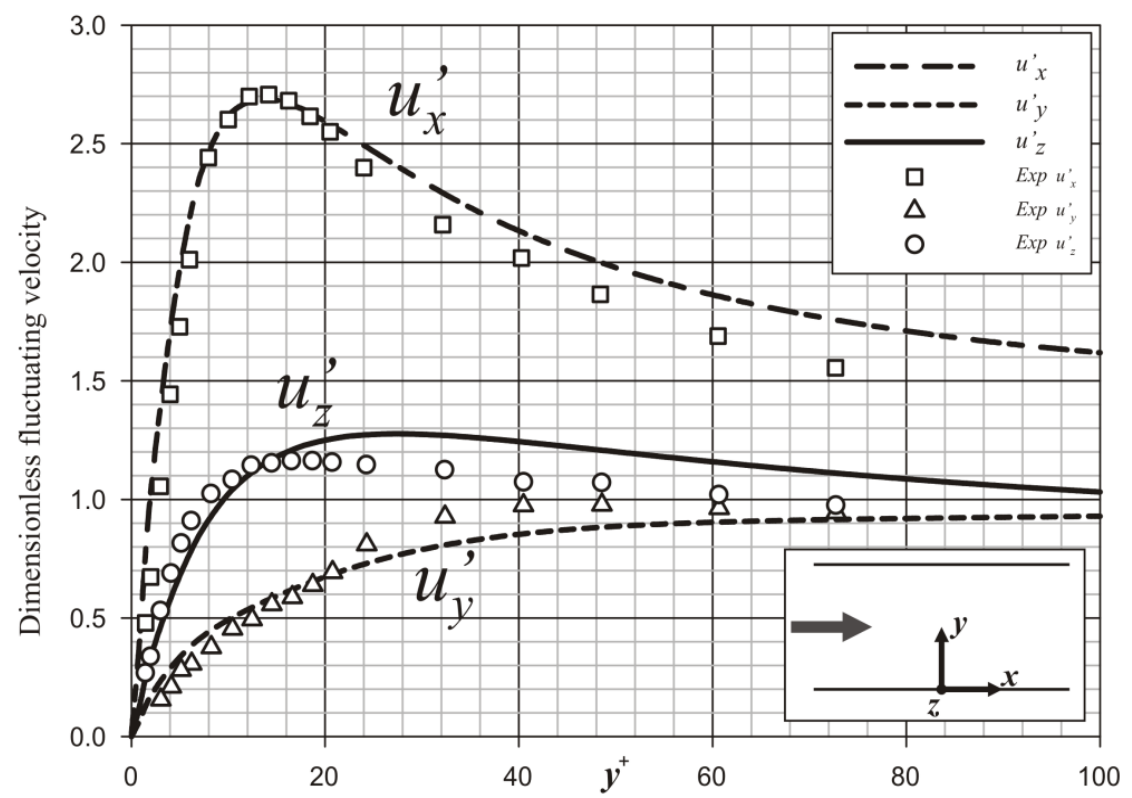

Figure 2: $\quad$ Experimental versus RSM simulation results. 


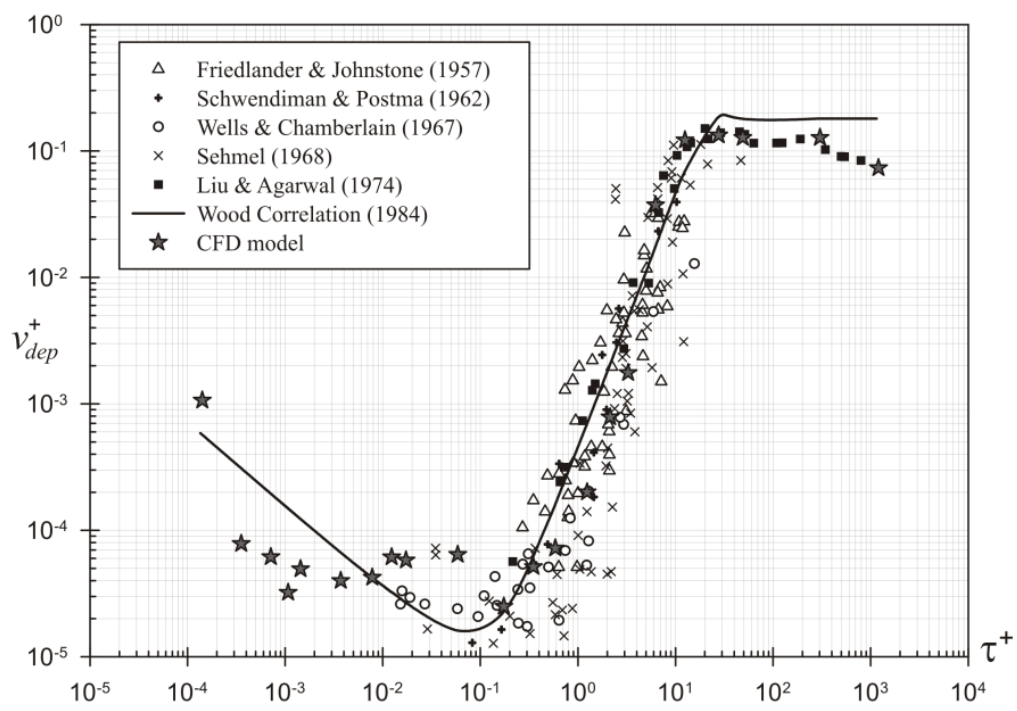

Figure 3: Evaluation of Brownian effect.

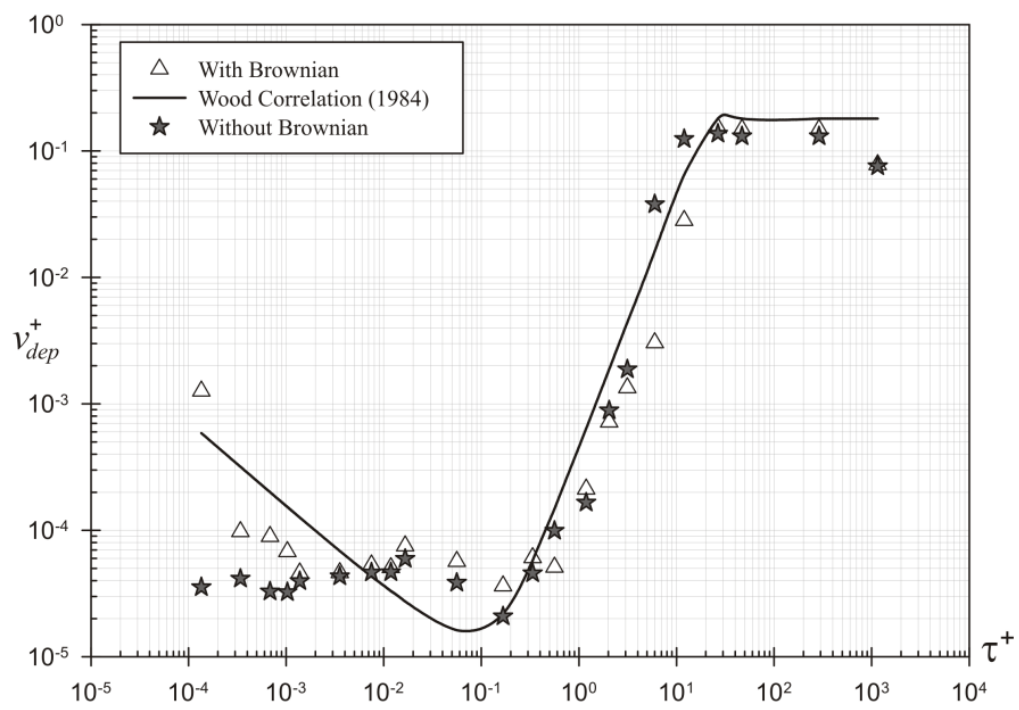

Figure 4: Experimental versus simulation results.

\subsection{Dispersed phase}

With the proposed methodology, information about temporal evolution of particle deposited mass and number of attempts was stored. This data was used to obtain the dimensionless deposition velocity, $\mathrm{v}_{\mathrm{d}}^{+}$. If $\mathrm{v}_{\mathrm{d}}^{+}$is plotted as a function 
of dimensionless particle relaxation time $\tau^{+}$, the results fall into the three deposition regimes. As shown in Figure 3, several previous experimental data and empirical correlations are compared with the present computational model showing a good agreement.

Also, the influence of the Brownian force was evaluated by enabling and disenabling this mechanism. For instance the Brownian effect is clearly shown in Figure 4 where $10^{-4}<\tau^{+}<10^{-1}$, which would represent submicron particles, the deposition velocity increases considerably when this mechanism is active.

\section{Conclusions}

A simple Lagrangian simulation CFD methodology, including turbulence effects has been tested with promising results. The CFD model results have shown good agreement with experimental tests and previous computational models published by other authors, so the simplifications proposed are acceptable. Moreover the methodology used allows the development of a more complex fouling model with a feasible computational cost.

The detail provided by the model demonstrates that Brownian diffusion is the main deposition mechanism in submicron particles for diluted isothermal process.

The particle size range simulated was big enough to cover all deposition regimes, and completely characterize the particle deposition process. The interaction of particles with turbulent eddies has proved to have a decisive effect on the particle deposition results measured, and the proposed methodology, with an appropriate model in the continuous phase and eddy life time submodel in the dispersed phase, is precise enough.

\section{References}

[1] Grillot J. M. \& Icart G. Fouling of a Cylindrical Probe and a Finned tube bundle in a diesel exhaust environment, Experimental Thermal and Fluid Science (14), pp. 442-454, (1997)

[2] Crowe M., Sommerfield C. and Tsuji Y. Multiphase Flows with Droplets and Particles. CRC Press, (1998)

[3] Fuchs N. A. The Mechanics of Aerosols. Pergamon Press (1964)

[4] Liu B. Y. H. and Agarwal J K. Experimental observation of aerosol deposition in turbulent flow. Aerosol Science, (5), pp. 145-155, (1974)

[5] Wood N.B. A simple method for the calculation of turbulent deposition to smooth and rough surfaces. Aerosol Science, 1(12), pp. 275-290, (1981)

[6] Hinds W. C. Aerosols an Industrial and Environmental Science, Academic Press New York (1982)

[7] Papavergos P. and Hedley A. B. Particle deposition behaviour from turbulent flows. Chemical Engineering Research and Design, 1(62), pp. 275-295, (1984) 
[8] Epstein N. Elements of particle deposition onto nonporous solid surfaces parallel to suspension flows. Experimental Thermal and Fluid Science, 1(14), pp.323-324, (1997)

[9] ANSYS-Fluent users guide (2007) \& UDF Manual. (2006)

[10] Tian L. \& Ahmadi G. Particle deposition in duct flows- Comparisons of different model predictions, (38), pp. 377-397, (2007)

[11] Morsi S.A. \& Alexander A.J. An investigation of particle trajectories in two-phase flow systems. J. Fluid Mech., 2(55), pp. 193-208, (1972).

[12] Saffman, P. G. The lift on a small sphere in a slow shear flow. Journal of Fluid Mechanics (22), pp. 385-400, (1965)

[13] Li A. \& Ahmadi G. (1992). Dispersion and deposition of spherical particles form point sources in a turbulent channel flow. Aerosol Science and Technology (16), 209-226, (1992)

[14] Narayanan C., Lakehal D., Botto L., and Soldati A. Mechanisms of particle deposition in a fully developed turbulent open channel flow. Physics of Fluids, 15(3), pp. 763-775, 2003. 